\title{
A CPW-FED SLOT-LOOP ANTENNA WITH LOW CROSS-POLARIZATION RADIATION
}

\author{
Shih-Wen Lu, Chih-Hsiang Yang, and Powen Hsu \\ Dept. of Electrical Engineering and Graduate Institute of Communication Engineering \\ National Taiwan University, Taipei 10617, Taiwan, R.O.C. \\ E-mail: phsu@cc.ee.ntu.edu.tw
}

\begin{abstract}
A method for reducing the level of cross-polarization radiation of the CPW-fed slot-loop antenna is presented. The method uses four small rectangular patches attached to the side of the substrate opposite to that containing the slot-loop and the CPW. Experimental results show that, with these shielding patches, the level of cross-polarization radiation of the antenna can be reduced to $\quad \sim-17 \mathrm{~dB}$ of that of the co-polarization radiation.
\end{abstract}

\section{Introduction}

The coplanar waveguide (CPW) fed rectangular slot-loop antenna is attractive and has been used in many microwave and millimeter-wave applications [1]-[5]. This is because that it has wide bandwidth and that its uniplanar structure provides an easy integration with the active and passive devices. This antenna, however, has a major disadvantage, i.e., its level of crosspolarization radiation is very high as compared to that of the co-polarization radiation. The cross-polarization arises mainly from the two slots parallel to the CPW line in the slot-loop. The longer the parallel slot, the higher the cross-polarization level [3]. In this paper, we propose a patch-shielded design of the CPW-fed slot-loop antenna which preserves the co-polarization radiation but significantly reduces the cross-polarization level.

\section{Antenna design}

The configuration of the patch shielded CPW-fed rectangular slot-loop antenna is shown in Fig. 1. Four small rectangular patches are placed on one side of the substrate, while a rectangular slot-loop fed by a CPW is arranged opposite to the patches in the ground plane on the other side of the substrate. The four small patches are placed to cover the four ends of the two slots in the slot-loop parallel to the CPW line, and each patch is placed centrally about the central line of the slot. The design frequency is chosen at the lowest frequency mode when the total slot-loop circumference is approximately $\lambda_{\mathrm{g}}$, where $\lambda_{\mathrm{g}}$ is the quasi-static guide wavelength given by $\lambda_{\mathrm{g}}=\lambda_{0} / \sqrt{\varepsilon_{\text {eff }}}$. Experimental models were fabricated on a low cost FR-4 substrate with $\varepsilon_{r}=4.3, \tan \delta=0.02$, and $\mathrm{d}=\mathbf{1 . 5 8} \mathrm{mm}$. Before attaching the shielding patches, the slot-loop is designed to be resonant at $2.4 \mathrm{GHz}$. The design parameters used are $L_{\mathrm{s} 1}=42 \mathrm{~mm}, L_{\mathrm{s} 2}=37 \mathrm{~mm}$, $O_{\mathrm{s} 1}=3.5 \mathrm{~mm}$, and $O_{\mathrm{s} 2}=1 \mathrm{~mm}$. The widths of the $C P W$ are $G=4 \mathrm{~mm}$ and $S=0.4 \mathrm{~mm}$, which correspond to a characteristic impedance of $50 \Omega$. The length and width of the four rectangular patches are $L_{p}=21 \mathrm{~mm}$ and $W_{p}=14 \mathrm{~mm}$, respectively.

\section{Results and discussion}

The simulation results were obtained by using the software package IE3D, which solves for the electric current on a metallic structure or the magnetic current in an aperture using the method of moments. To verify our simulation, experiments were carefully fabricated using the above parameters. Reflection coefficients of the antenna were measured by using an HP8510B network analyzer and adopting the TRL (through-reflect-line) calibration technique, and the measurements were referenced to the end of the CPW line (or the input of the slot-loop). Fig. 2 shows the reflection coefficients $S_{11}$ of the antenna. The agreement between simulation and 
experiment is excellent. It should be noted that, without the four patches, the slot-loop was resonant at $2.4 \mathrm{GHz}$, while after attaching the four patches, the resonant frequency is lowered to $1.93 \mathrm{GHz}$. This is because that the effective permittivity $\varepsilon_{\text {eff }}$ with the patches is larger than that without the patches. The radiation patterns of the antenna were measured in an anechoic chamber. The measured H-plane (xz-plane) patterns of both co-polarization and crosspolarization are shown in Fig. 3. For comparison, the cross-polarization pattern of the antenna without the patches is also shown in this figure. It is seen that the cross-polarization level is significantly reduced when the shielding patches are attached. The cross-polarization level is now only $\sim-17 \mathrm{~dB}$ as compared to that of the co-polarization.

\section{References}

[1] G. Forma and J. M. Laheurte, "Compact oscillating slot loop antenna with conductor backing”, Electron. Lett., vol. 32, no. 18, pp. 1633-1635, 1996.

[2] T. M. Weller, L. P. Katehi, and G. M. Rebeiz, "Single and double folded-slot antennas on semi-infinite substrates", IEEE Trans. Antennas Propagat., vol. 43, no. 12, pp. 1423-1428, Dec. 1995.

[3] H. S. Tsai and R. A. York, "FDTD analysis of CPW-fed folded-slot and multiple-slot antenna on thin substrate", IEEE Trans. Antennas Propagat., vol. 44, no. 2, pp. 217-226, Feb. 1996.

[4] G. P. Gauthier, S. Raman, and G. M. Rebeiz, "A 90-100 GHz double-folded slot antenna", IEEE Trans. Antennas Propagat., vol. 47, no. 6, pp. 1120-1122, Jun. 1999.

[5] H. C. Liu, T. S. Horng, and N. G. Alexopoulos, "Radiation of printed antennas with a coplanar waveguide feed", IEEE Trans. Antennas Propagat., vol. 43, no. 10, pp. 1143-1148, Oct. 1995.

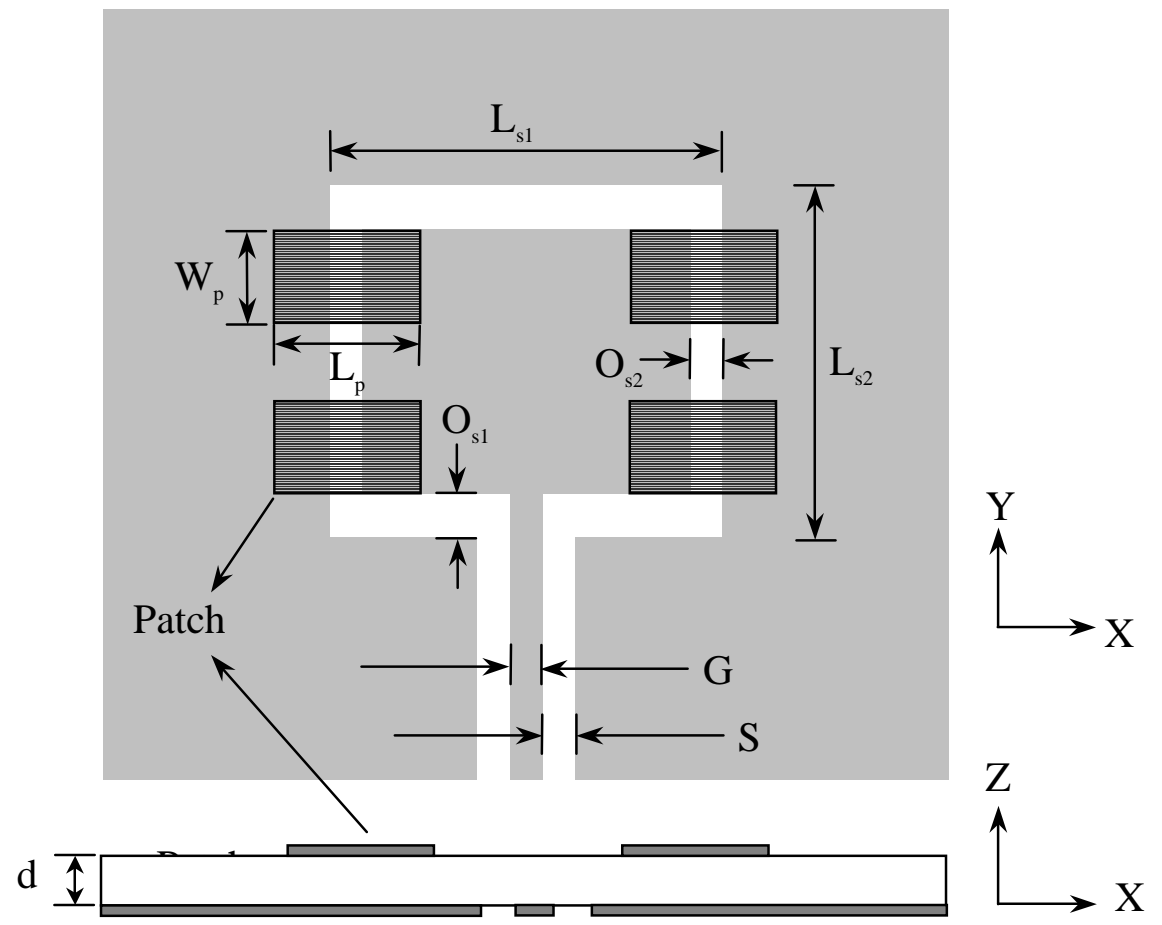

Fig. 1 Configuration of patch shielded CPW-fed slot-loop antenna 

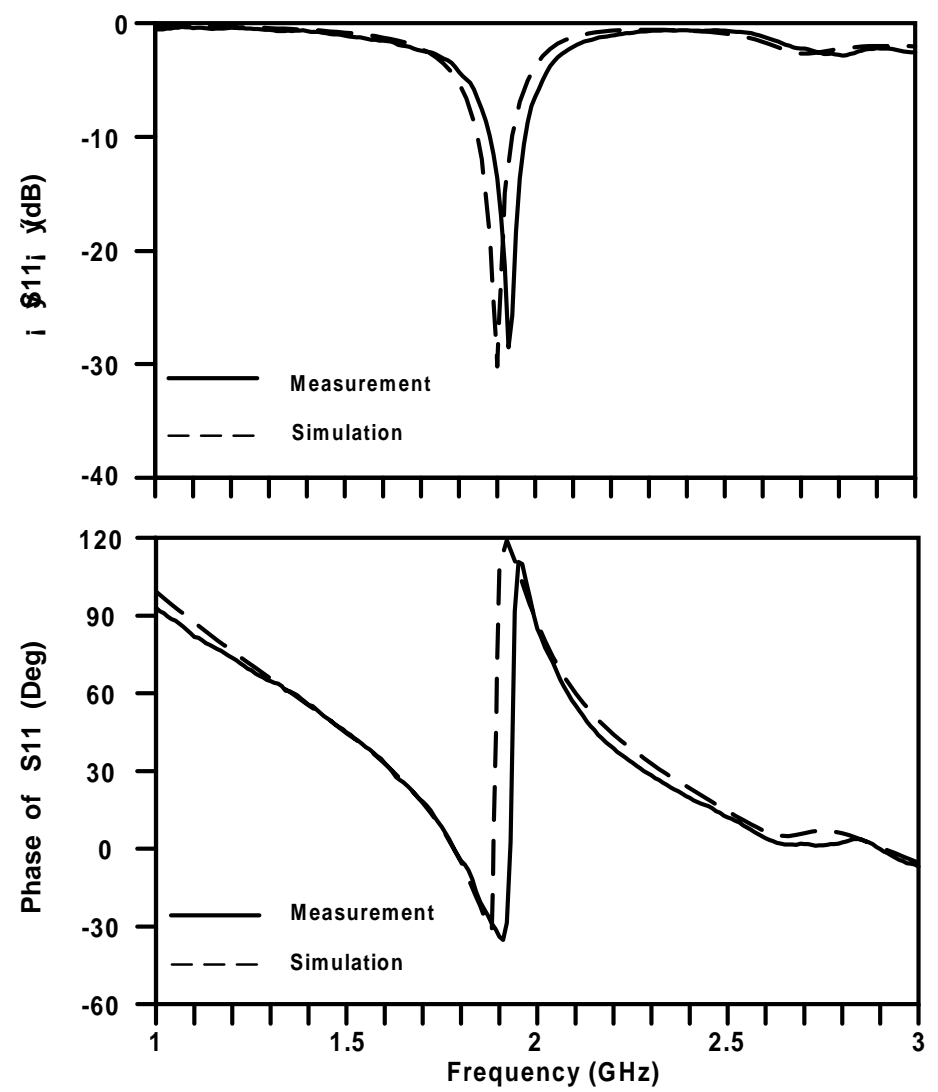

Fig. 2 Reflection coefficient of patch shielded CPW-fed slot-loop antenna

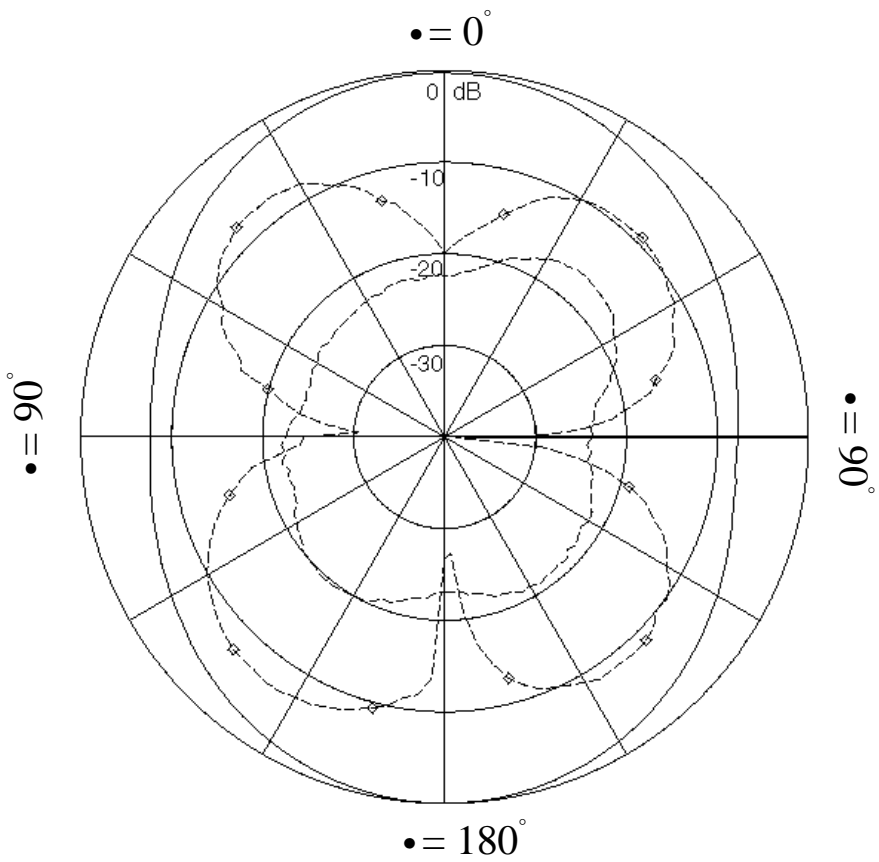

Fig. 3 Measured H-plane pattern of patch shielded CPW-fed slot-loop antenna

Co-polarization (With patch)

Cross-polarization (With patch)

$\checkmark \checkmark$ Cross-polarization (Without patch) 